\title{
HOCHSCHILD COMOHOLOGY FOR ALGEBRAS OF DIHEDRAL TYPE, VI. THE FAMILY $D(2 \mathcal{B})(k, s, 1)$
}

\author{
A. I. GENERALOV AND D. B. ROMANOVA \\ Dedicated to the 70th anniversary \\ of Serge Vladimirovich Vostokov, \\ a remarkable mathematician and bright personality
}

\begin{abstract}
The Hochschild cohomology groups are calculated for algebras of dihedral type in the series $D(2 \mathcal{B})(k, s, c)$ (in accordance with K. Erdmann's classification) in the case where the parameter $c \in K$ occurring in the defining relations for this series equals 1 . The calculations involve the bimodule resolvent for the algebras of this type, which is also constructed in the present paper. The results are applied to refine Erdmann's classification, specifically, it is proved that algebras corresponding to different values of $c$ represent different classes of derived equivalence, and, in particular, different classes of Morita-equivalence.
\end{abstract}

\section{$\S 1$. INTRODUCTION}

The present paper is devoted to calculation of the Hochschild cohomology groups for algebras of dihedral type contained in the family $D(2 \mathcal{B})$. The algebras of this family are presented in the famous K. Erdmann's classification [1, but it was unknown whether the algebras corresponding to different values of the parameter $c$ represent different classes of Morita-equivalence for algebras studied by K. Erdmann. A similar problem for classes of derived equivalence for those algebras was also open (see [2]).

The parameter $c$ mentioned above (involved in the defining relations of the algebras in $D(2 \mathcal{B})$ ) takes two values: 0 and 1 . Combining the results of the present paper on calculation of the Hochschild cohomology groups for the case of $c=1$ with similar results in [3] (for $c=0$ ), we give, in particular, the answer to the above problems. Indeed, the algebras in the family $D(2 \mathcal{B})$ that correspond to different values of $c$ are not derived equivalent (and hence, are not Morita-equivalent). Notice that, even for "two-vertices" group blocks with the dihedral defect group, the dilemma $c=0$ or $c=1$ has not been studied completely (see, e.g., 4]). Hence, the calculation of the Hochschild cohomology groups for the family $D(2 \mathcal{B})$ fulfilled in $[3$ and in the present paper will perhaps find an application in the study of such group blocks.

As before, we use the approach of the paper [5], in which a description of the Hochschild cohomology algebra was given for the algebras of dihedral type in the family $D(3 \mathcal{K})$ over an algebraically closed ground field of characteristic two (again we use the notation of [1]). This approach can be outlined as follows: on the basis of some empirical calculation, we construct the minimal projective bimodule resolution for the algebras under consideration, and then the Hochschild cohomology groups and the multiplication in the cohomology algebra are calculated with the help of this resolution.

2010 Mathematics Subject Classification. Primary 16E40.

Key words and phrases. Hochschild cohomology groups, algebras of dihedral type, bimodule resolvent.

The first author was supported by RFBR (grant no. 13-01-00902). 
Earlier, this approach was applied to several other families of algebras of dihedral type (see [6, 7, 8] ). Similarly, the Hochschild cohomology was studied for several families of algebras of semidihedral type in [9, 10, 11, 12, and for families of algebras of quaternion type in $13,14,15,16$.

There are results concerning the description of the algebra $\mathrm{HH}^{*}(R)$ that were obtained for the so-called Möbius algebra (see [17, 18, 19, 20]) and for selfinjective algebras of finite representation type with tree class $D_{n}$ (see [21, 22, 23, 24, 25, 26, 27]), and for a family of selfinjective algebras of finite representation type with tree class $E_{6}$ (see [28]). Also, the approach of [5] was applied for the calculation of the algebra $\mathrm{HH}^{*}(R)$ for Liu-Shulz algebras (see 29]).

We describe the organization of the paper briefly. In $\S 2$, we formulate the main result, Theorem 2.1, in which the Hochschild cohomology groups are described for the family of algebras of dihedral type mentioned above. In $\S 3$, the minimal projective resolution is constructed for the algebra $R$ viewed as a module over its enveloping algebra $\Lambda=R^{\mathrm{e}}=R \otimes_{K} R^{\mathrm{op}}$. Finally, in $\S 4$ we use this resolution to calculate the groups $\operatorname{HH}^{n}(R)$ for the algebras under consideration. The results of our calculation can be applied to group blocks that have the dihedral defect group and two simple modules. Earlier, the Hochschild cohomology groups were calculated for group blocks with the dihedral defect group only in the case of blocks with one or three simple modules; see [30].

\section{§2. Statement of the MAin Result}

Let $K$ be an algebraically closed field with an arbitrary characteristic, $R$ a finitedimensional $K$-algebra, $\Lambda=R^{\mathrm{e}}=R \otimes_{K} R^{\mathrm{op}}$ its the enveloping algebra, $\operatorname{HH}^{n}(R)=$ $\operatorname{Ext}_{\Lambda}^{n}(R, R)$ the $n$th Hochschild cohomology group of the algebra $R$ (with coefficients in the $R$-bimodule $R$ ). Hence, if $P_{\bullet} \rightarrow R$ is a $\Lambda$-projective resolution of the algebra $R$, then $\mathrm{HH}^{n}(R)=\mathrm{H}^{n}\left(\operatorname{Hom}_{\Lambda}\left(P_{\bullet}, R\right)\right)$.

The algebras $R_{k, s, c}$ that form the family $D(2 \mathcal{B})(k, s, c)$ (over an algebraically closed field $K$ with an arbitrary characteristic) are defined by the following quiver with relations

$$
\begin{gathered}
Q^{(\mathcal{B})}: \\
\beta \gamma=\eta \beta=\gamma \eta=0, \quad(\gamma \beta \alpha)^{k}=(\alpha \gamma \beta)^{k}, \\
\alpha^{2}=c(\gamma \beta \alpha)^{k}, \quad \eta^{s}=(\beta \alpha \gamma)^{k},
\end{gathered}
$$$$
\text { where } k, s \in \mathbb{N}, s \geq 2 \text {, and } c \in\{0,1\} \text { (composition is written from right to left). }
$$

Note that if char $K \neq 2$, then we may assume that $c=0$. Since the groups $\operatorname{HH}^{n}(R)$ for the algebras $R=R_{k, s, 0}$ were calculated in [3], we always assume that the parameter $c$ equals 1 and that char $K=2$.

The maim result of the paper is the following description of the Hochschild cohomology groups for the family of algebras under consideration.

Theorem 2.1. Put $R=R_{k, s, 1}$, where $k, s \in \mathbb{N}, s \geq 2$. Then the dimensions of the groups $\mathrm{HH}^{n}(R)$ are described as follows:

$$
\begin{aligned}
& \operatorname{dim}_{K} \mathrm{HH}^{0}(R)=k+s+2 ; \\
& \operatorname{dim}_{K} \mathrm{HH}^{1}(R)= \begin{cases}k+s+3 & \text { if } k \text { and } s \text { are both even, } \\
k+s+2 & \text { if exactly one number among } k \text { and } s \text { is even, } \\
k+s+1 & \text { if } k \text { and } s \text { are both odd; }\end{cases}
\end{aligned}
$$


(c) $\operatorname{dim}_{K} \mathrm{HH}^{2}(R)= \begin{cases}k+s+4 & \text { if } k \text { and } s \text { are both even, } \\ k+s+3 & \text { if exactly one number among } k \text { and } s \text { is even, } \\ k+s+2 & \text { if } k \text { and } s \text { are odd; }\end{cases}$

(d) $\operatorname{dim}_{K} \operatorname{HH}^{3}(R)= \begin{cases}k+s+5 & \text { if } k \text { and } s \text { are even, } \\ k+s+4 & \text { otherwise; }\end{cases}$

(e) $\operatorname{dim}_{K} \operatorname{HH}^{4}(R)= \begin{cases}k+s+6 & \text { if } k \text { and } s \text { are even, } \\ k+s+5 & \text { otherwise; }\end{cases}$

(f) for any $n \geq 5$,

$\operatorname{dim}_{K} \mathrm{HH}^{n}(R)-\operatorname{dim}_{K} \mathrm{HH}^{n-4}(R)=\left\{\begin{array}{ll}4 & \text { if } n \equiv 1 \\ 5 & \text { otherwise }\end{array}(\bmod 3)\right.$,

Corollary 2.2. Put $R=R_{k, s, 1}$. Then

$$
\operatorname{dim}_{K} \mathrm{HH}^{n}(R)-\operatorname{dim}_{K} \mathrm{HH}^{n-12}(R)=14 .
$$

The above corollary and [3, Corollary 2.2] imply the following result, which completes K. Erdmann's classification in [1].

Corollary 2.3. Let char $K=2$, and let $k, s \in \mathbb{N}, s \geq 2$. Then the algebras $R_{k, s, 0}$ and $R_{k, s, 1}$ are not derived equivalent, and hence, are not Morita-equivalent.

Remark 2.4. The statement in Corollary 2.3 can be deduced also from the comparison of dimensions of the groups $\mathrm{HH}^{1}(R)$ for $R=R_{k, s, 0}$ and $R=R_{k, s, 1}$, respectively; see Theorem 2.1 (b) and [3, Theorem 2.1 (IIa)].

\section{§3. Resolution}

Let $R=R_{k, s, 1}$ be the algebra defined in $\oint_{2}$, We denote by $e_{i}, i=0,1$, the idempotents of the algebra $R$ that correspond to the vertices of the quiver $Q^{(\mathcal{B})}$. Then the modules

$$
P_{i j}=\Lambda\left(e_{i} \otimes e_{j}\right), \quad i, j \in\{0,1\},
$$

form a complete set of representatives of principal indecomposable left $\Lambda$-modules where $\Lambda=R^{\mathrm{e}}$.

Multiplication from the right by an element $w \in \Lambda$ induces an endomorphism $w^{*}$ of the left $\Lambda$-module $\Lambda$, and moreover, if $w \in\left(e_{i} \otimes e_{j}\right) \Lambda\left(e_{k} \otimes e_{l}\right)$, then $w^{*}$ induces a homomorphism $w^{*}: P_{i j} \rightarrow P_{k l}$. For simplicity, this homomorphism of multiplication (from the right) by $w \in \Lambda$ will be denoted also by $w$.

We introduce an abbreviated notation for several elements of the algebra $R$ :

$$
a:=\alpha \gamma \beta, \quad b:=\beta \alpha \gamma, \quad g:=\gamma \beta \alpha .
$$

The set

$$
\mathcal{B}=\mathcal{B}_{00} \cup \mathcal{B}_{10} \cup \mathcal{B}_{01} \cup \mathcal{B}_{11}
$$

where

$$
\begin{aligned}
& \mathcal{B}_{00}=\left\{a^{i+1}, g^{i}, \gamma \beta a^{i}, \alpha g^{i} \mid 0 \leq i \leq k-1\right\}, \\
& \mathcal{B}_{10}=\left\{\beta a^{i}, \beta \alpha g^{i} \mid 0 \leq i \leq k-1\right\}, \\
& \mathcal{B}_{01}=\left\{\gamma b^{i}, \alpha \gamma b^{i} \mid 0 \leq i \leq k-1\right\}, \\
& \mathcal{B}_{11}=\left\{\eta^{i} \mid 0 \leq i \leq s\right\} \cup\left\{b^{i} \mid 1 \leq i \leq k-1\right\},
\end{aligned}
$$


is called the standard basis of the algebra $R$.

We consider the following projective $\Lambda$-modules:

$$
\begin{aligned}
& L_{0}=P_{00} \oplus P_{11}, \\
& L_{1}=\left(P_{00} \oplus P_{10}\right) \oplus\left(P_{11} \oplus P_{01}\right), \\
& L_{2}=P_{00}^{2} \oplus P_{11}^{2}, \\
& L_{3}=\left(P_{00} \oplus P_{10}\right) \oplus\left(P_{01} \oplus P_{11}\right),
\end{aligned}
$$

and then use these modules to construct the following diagram $\mathcal{B}_{\bullet \bullet}$ (its rows and columns are numbered by $0,1,2, \ldots)$ :

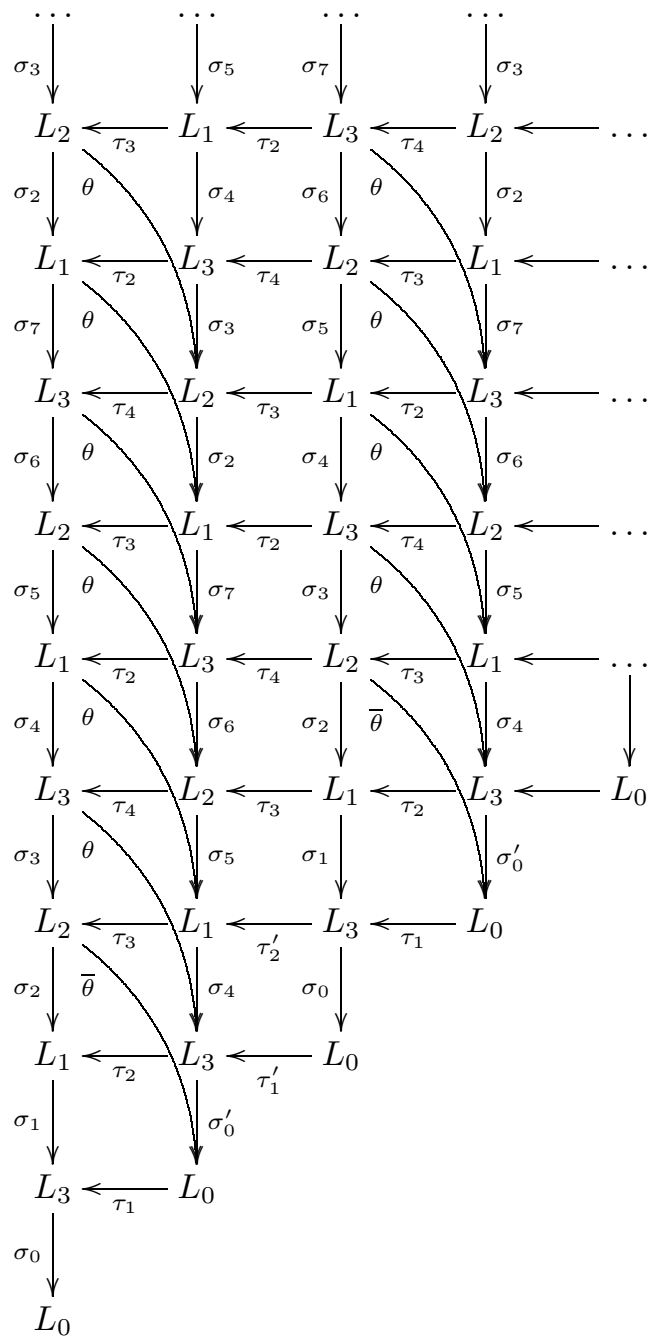

In this diagram, the homomorphisms are described with the help of matrices corresponding to the direct decompositions of modules in (3.2) (it is clear that $L_{1} \simeq L_{3}$, but the fixed orders of direct summands are important for us):

$$
\sigma_{0}=\left(\begin{array}{cccc}
e_{0} \otimes \alpha+\alpha \otimes e_{0} & \beta \otimes e_{0} & e_{0} \otimes \gamma & 0 \\
0 & e_{1} \otimes \beta & \gamma \otimes e_{1} & e_{1} \otimes \eta+\eta \otimes e_{1}
\end{array}\right)
$$




$$
\sigma_{1}=\left(\begin{array}{cccc}
e_{0} \otimes \alpha+\alpha \otimes e_{0}+\sum_{i=0}^{k-1} \gamma \beta a^{i} \otimes g^{k-1-i} & 0 & 0 & 0 \\
\sum_{i=0}^{k-1} \gamma b^{i} \otimes \alpha g^{k-1-i} & \eta \otimes e_{0} & e_{1} \otimes \gamma & 0 \\
\sum_{i=0}^{k-1} g^{i} \otimes \beta \alpha g^{k-1-i} & 0 & \beta \otimes e_{1} & e_{0} \otimes \eta \\
0 & e_{1} \otimes \beta & 0 & \gamma \otimes e_{1}
\end{array}\right)
$$$$
\sigma_{2}=\left(\begin{array}{cccc}
e_{0} \otimes \alpha+\alpha \otimes e_{0} & 0 & 0 & 0 \\
0 & \gamma \otimes e_{0} & e_{1} \otimes \gamma & 0 \\
0 & 0 & \eta \otimes e_{1} & e_{1} \otimes \eta \\
0 & e_{0} \otimes \beta & 0 & \beta \otimes e_{1}
\end{array}\right)
$$$$
\sigma_{3}=\left(\begin{array}{cccc}
e_{0} \otimes \alpha+\alpha \otimes e_{0}+\gamma \beta a^{k-1} \otimes e_{0} & 0 & 0 & 0 \\
0 & \beta \otimes e_{0} & e_{0} \otimes \gamma & 0 \\
0 & 0 & \gamma \otimes e_{1} & e_{1} \otimes \eta \\
0 & e_{1} \otimes \beta & 0 & \eta \otimes e_{1}
\end{array}\right),
$$

$$
\sigma_{4}=\left(\begin{array}{cccc}
e_{0} \otimes \alpha+\alpha \otimes e_{0} & 0 & 0 & 0 \\
0 & \eta \otimes e_{0} & e_{1} \otimes \gamma & 0 \\
0 & 0 & \beta \otimes e_{1} & e_{0} \otimes \eta \\
0 & e_{1} \otimes \beta & 0 & \gamma \otimes e_{1}
\end{array}\right),
$$

$$
\begin{aligned}
& \sigma_{5}=\left(\begin{array}{cccc}
e_{0} \otimes \alpha+\alpha \otimes e_{0}+\alpha \otimes e_{0}+\gamma \beta a^{k-1} & 0 & 0 & 0 \\
0 & \gamma \otimes e_{0} & e_{1} \otimes \gamma & 0 \\
0 & 0 & \eta \otimes e_{1} & e_{1} \otimes \eta \\
0 & e_{0} \otimes \beta & 0 & \beta \otimes e_{1}
\end{array}\right) \text {, } \\
& \sigma_{6}=\left(\begin{array}{cccc}
e_{0} \otimes \alpha+\alpha \otimes e_{0} & 0 & 0 & 0 \\
0 & \gamma \otimes e_{0} & e_{1} \otimes \gamma & 0 \\
0 & 0 & \eta \otimes e_{1} & e_{1} \otimes \eta \\
0 & e_{0} \otimes \beta & 0 & \beta \otimes e_{1}
\end{array}\right),
\end{aligned}
$$

$$
\sigma_{7}=\left(\begin{array}{cccc}
e_{0} \otimes \alpha+\alpha \otimes e_{0}+\gamma \beta a^{k-1} & 0 & 0 & 0 \\
0 & \beta \otimes e_{0} & e_{0} \otimes \gamma & 0 \\
0 & 0 & \gamma \otimes e_{1} & e_{1} \otimes \eta \\
0 & e_{1} \otimes \beta & 0 & \eta \otimes e_{1}
\end{array}\right)
$$

$$
\sigma_{0}^{\prime}=\left(\begin{array}{cccc}
e_{0} \otimes \alpha+\alpha \otimes e_{0}+\gamma \beta a^{k-1} & \beta \otimes e_{0} & e_{0} \otimes \gamma & 0 \\
0 & e_{1} \otimes \beta & \gamma \otimes e_{1} & e_{1} \otimes \eta+\eta \otimes e_{1}
\end{array}\right)
$$$$
\tau_{1}=\left(\begin{array}{cc}
\sum_{i=0}^{k-1}\left(\gamma \beta a^{i} \otimes g^{k-1-i}+a^{i} \otimes \gamma \beta a^{k-1-i}\right) & \sum_{i=0}^{k-1} \beta a^{i} \otimes \gamma b^{k-1-i} \\
\sum_{i=0}^{k-1}\left(\gamma b^{i} \otimes \alpha g^{k-1-i}+\alpha \gamma b^{i} \otimes a^{k-1-i}\right) & \sum_{i=0}^{k-1} b^{i} \otimes \alpha \gamma b^{k-1-i} \\
\sum_{i=0}^{k-1}\left(g^{i} \otimes \beta \alpha g^{k-1-i}+\alpha g^{i} \otimes \beta a^{k-1-i}\right) & \sum_{i=0}^{k-1} \beta \alpha g^{i} \otimes b^{k-1-i} \\
0 & \sum_{j=0}^{s-1} \eta^{j} \otimes \eta^{s-1-j}
\end{array}\right),
$$

$$
\tau_{2}=\left(\begin{array}{cccc}
e_{0} \otimes \gamma \beta a^{k-1}+\gamma \beta a^{k-1} \otimes e_{0} & 0 & 0 & 0 \\
0 & \eta^{s-1} \otimes e_{0} & 0 & e_{1} \otimes \alpha \gamma b^{k-1} \\
0 & e_{1} \otimes \beta \alpha g^{k-1} & \alpha \gamma b^{k-1} \otimes e_{1} & 0 \\
0 & 0 & e_{0} \otimes \eta^{s-1} & \beta \alpha g^{k-1} \otimes e_{1}
\end{array}\right),
$$$$
\tau_{3}=\left(\begin{array}{cccc}
e_{0} \otimes \gamma \beta a^{k-1}+\gamma \beta a^{k-1} \otimes e_{0} & 0 & 0 & 0 \\
0 & \beta \alpha g^{k-1} \otimes e_{0} & 0 & e_{0} \otimes \alpha \gamma b^{k-1} \\
0 & e_{1} \otimes \beta \alpha g^{k-1} & \eta^{s-1} \otimes e_{1} & 0 \\
0 & 0 & e_{1} \otimes \eta^{s-1} & \alpha \gamma b^{k-1} \otimes e_{1}
\end{array}\right),
$$ 


$$
\begin{aligned}
\tau_{4} & =\left(\begin{array}{cccc}
e_{0} \otimes \gamma \beta a^{k-1}+\gamma \beta a^{k-1} \otimes e_{0} & 0 & 0 & 0 \\
0 & \alpha \gamma b^{k-1} \otimes e_{0} & 0 & e_{1} \otimes \alpha \gamma b^{k-1} \\
0 & e_{0} \otimes \beta \alpha g^{k-1} & \beta \alpha g^{k-1} \otimes e_{1} & 0 \\
0 & 0 & e_{1} \otimes \eta^{s-1} & \eta^{s-1} \otimes e_{1}
\end{array}\right), \\
\tau_{1}^{\prime} & =\left(\begin{array}{cc}
\sum_{i=0}^{k-1}\left(\gamma \beta a^{i} \otimes g^{k-1-i}+a^{i} \otimes \gamma \beta a^{k-1-i}\right) & \sum_{i=0}^{k-1} \beta a^{i} \otimes \gamma b^{k-1-i} \\
* & \sum_{i=0}^{k-1} b^{i} \otimes \alpha \gamma b^{k-1-i} \\
\sum_{i=0}^{k-1}\left(g^{i} \otimes \beta \alpha g^{k-1-i}+\alpha g^{i} \otimes \beta a^{k-1-i}\right) & \sum_{i=0}^{k-1} \beta \alpha g^{i} \otimes b^{k-1-i} \\
0 & \sum_{j=0}^{s-1} \eta^{j} \otimes \eta^{s-1-j}
\end{array}\right),
\end{aligned}
$$

where

$$
\begin{aligned}
\left(\tau_{1}^{\prime}\right)_{21} & =\sum_{i=0}^{k-1}\left(\gamma b^{i} \otimes \alpha g^{k-1-i}+\alpha \gamma b^{i} \otimes a^{k-1-i}\right)+\gamma b^{k-1} \otimes \gamma \beta a^{k-1}, \\
\tau_{2}^{\prime} & =\left(\begin{array}{cccc}
e_{0} \otimes \gamma \beta a^{k-1}+\gamma \beta a^{k-1} \otimes e_{0} & 0 & 0 & 0 \\
0 & \eta^{s-1} \otimes e_{0} & 0 & e_{1} \otimes \alpha \gamma b^{k-1} \\
\alpha \gamma b^{k-1} \otimes \beta a^{k-1} & e_{1} \otimes \beta \alpha g^{k-1} & +\alpha \gamma b^{k-1} \otimes e_{1} & 0 \\
0 & 0 & e_{0} \otimes \eta^{s-1} & \beta \alpha g^{k-1} \otimes e_{1}
\end{array}\right), \\
\theta & =\left(\begin{array}{cccc}
e_{0} \otimes \alpha & 0 & 0 & 0 \\
0 & 0 & 0 & 0 \\
0 & 0 & 0 & 0 \\
0 & 0 & 0 & 0
\end{array}\right), \bar{\theta}=\left(\begin{array}{cccc}
e_{0} \otimes \alpha & 0 & 0 & 0 \\
0 & 0 & 0 & 0
\end{array}\right) .
\end{aligned}
$$

Observe that the above diagram commutes, and every row (every column, respectively) of it is a complex. Moreover, we have $\sigma_{0} \tau_{1}=0$ and $\sigma_{0}^{\prime} \tau_{1}=0$. (These facts are verified by direct calculation.) Applying the process similar to building the total complex of a bicomplex, we obtain the complex $\operatorname{Tot}\left(B_{\bullet \bullet}\right)$ (cf. [31]). As an augmentation map $\mu: B_{0,0}=P_{00} \oplus P_{11} \rightarrow R$ we take the map induced by multiplication in $R: \mu(r \otimes s)=r s$.

Theorem 3.1. Put $R=R_{k, s, 1}$, where $k, t \in \mathbb{N}, s \geq 2$. The "total complex" $Q_{\bullet}=$ $\left(Q_{n}, d_{n}^{Q}\right)=\operatorname{Tot}\left(B_{\bullet \bullet}\right)$ of the diagram $B_{\bullet \bullet}$ together with the augmentation map $\mu: Q_{0} \rightarrow R$ is a minimal $\Lambda$-projective resolution of the algebra $R$.

Remark 3.2. In order to illustrate the process of constructing the "total complex" $\operatorname{Tot}\left(B_{\bullet \bullet}\right)$, we write out (in the corresponding block form) several first differentials $d_{n}^{Q}$ :

$$
\begin{aligned}
& d_{0}^{Q}=\sigma_{0} ; \quad d_{1}^{Q}=\left[\begin{array}{ll}
\tau_{1} & \sigma_{1}
\end{array}\right] ; \quad d_{2}^{Q}=\left[\begin{array}{cc}
\sigma_{0}^{\prime} & \bar{\theta} \\
\tau_{2} & \sigma_{2}
\end{array}\right] ; \\
& d_{3}^{Q}=\left[\begin{array}{ccc}
\tau_{1}^{\prime} & \sigma_{4} & \theta \\
0 & \tau_{3} & \sigma_{3}
\end{array}\right] ; \quad d_{4}^{Q}=\left[\begin{array}{ccc}
\sigma_{0} & 0 & 0 \\
\tau_{2}^{\prime} & \sigma_{5} & \theta \\
0 & \tau_{4} & \sigma_{4}
\end{array}\right] ; \quad d_{5}^{Q}=\left[\begin{array}{cccc}
\tau_{1} & \sigma_{1} & 0 & 0 \\
0 & \tau_{3} & \sigma_{6} & \theta \\
0 & 0 & \tau_{2} & \sigma_{5}
\end{array}\right] .
\end{aligned}
$$

Proof of Theorem 3.1. A direct calculation shows that $Q \bullet$ is a complex and $\mu \cdot d_{0}^{Q}=0$. To prove that the resulting complex is exact, we use Theorem 1 in 32. It suffices to prove that, tensoring the complex $\mu: Q_{\bullet} \rightarrow R$ by the simple $R$-module $S_{i}$, we obtain the minimal projective resolution of the module $S_{i}$ (such resolutions of the modules $S_{i}$, $i=0,1$, were described in [33). This fact is verified by direct calculation. We leave this detailed verification to the reader.

Consider the diagram $\mathcal{A}_{\bullet} \bullet$ that consists of the first two columns in the diagram $\mathcal{B}_{\bullet \bullet}$ with numbers 0 and 1 (the remaining columns in $\mathcal{A}_{\bullet \bullet}$ are zero). Let $X_{\bullet}=\operatorname{Tot}\left(\mathcal{A}_{\bullet \bullet}\right)$ be the "total complex" of this diagram in the same sense as above. 
Proposition 3.3. There is a short exact sequence of complexes

$$
0 \rightarrow X_{\bullet} \stackrel{\imath}{\rightarrow} Q \bullet \stackrel{\pi}{\rightarrow} Q \bullet[-4] \rightarrow 0
$$

that splits in each degree.

Proof. This follows immediately from the construction of the diagram $\mathcal{B} .$.

\section{$\S 4$. Cohomology groups}

As before, let $R=R_{k, s, 1}$ be a $K$-algebra defined in 92 Assume char $K=2$. To calculate the cohomology $\mathrm{HH}^{n}(R)$ of the algebra $R$, we use the complex

$$
\left(\operatorname{Hom}_{\Lambda}\left(Q_{n}, R\right), \delta^{n}=\operatorname{Hom}_{\Lambda}\left(d_{n}^{Q}, R\right)\right)_{n \geq 0},
$$

where $\mu: Q_{\bullet} \rightarrow R$ is the bimodule resolution of the algebra $R$ described in $\$ 3$, In the sequel, if $f \in \operatorname{Ker} \delta^{n}$ is an $n$-cocycle, we keep the notation $f$ for its cohomology class cl $f \in \mathrm{HH}^{n}(R)$.

Remark 4.1. Since $P_{i j}=\Lambda \cdot\left(e_{i} \otimes e_{j}\right)$, any $\Lambda$-homomorphism $f: Q_{n} \rightarrow R$ is defined by its values at the corresponding generators $e_{i} \otimes e_{j}$ of the $P_{i j}$ that occur in the decomposition of the module $Q_{n}$; moreover, we have $f\left(e_{i} \otimes e_{j}\right) \in e_{i} R e_{j}$. In the sequel, we identify $f$ with this set of values, which is ordered by increasing of the second index in the decomposition $Q_{n}=\sum_{r+t=n} B_{r t}$ (and in accordance with the order of the modules in the decompositions (3.2) $)$. If a subsequence of this collection contains only zeros, say $r$ zeros, we denote such subsequence by $\mathrm{O}_{r}$. Similarly, the zero $(r \times t)$-matrix will be denoted by $\mathrm{O}_{r, t}$; moreover, we omit the indices that indicate the size of such a matrix if this size is obvious from the context. Furthermore, in the notation of (3.1), we have

$$
\operatorname{dim}_{K} \operatorname{Hom}\left(P_{i j}, R\right)=\left|\mathcal{B}_{i j}\right|, \quad i, j \in\{0,1\} .
$$

Remark 4.2. For a homomorphism $f=w^{*}: \Lambda \rightarrow \Lambda$ of multiplication from the right by $w \in \Lambda$, the induced homomorphism of Abelian groups

$$
\widetilde{w}: \operatorname{Hom}_{\Lambda}(f, R): \operatorname{Hom}_{\Lambda}(\Lambda, R) \simeq R \rightarrow \operatorname{Hom}_{\Lambda}(\Lambda, R) \simeq R
$$

acts as follows, in accordance with the identification mentioned above: $r \in R$ is mapped to $w * r$; here the symbol $*$ corresponds to the $\Lambda$-module structure on $R$.

Upon this identification, the differential

$$
\delta^{0}: \operatorname{Hom}_{\Lambda}\left(Q_{0}, R\right) \rightarrow \operatorname{Hom}_{\Lambda}\left(Q_{1}, R\right)
$$

is described by the following formula: for any $r_{i} \in e_{i} R e_{i}(i=0,1)$ we have

$$
\delta^{0}\left(r_{0}, r_{1}\right)=\left(\alpha r_{0}+r_{0} \alpha, \quad \beta r_{0}+r_{1} \beta, \quad r_{0} \gamma+\gamma r_{1}, \quad \eta r_{1}+r_{1} \eta\right) .
$$

Proposition 4.3. $\operatorname{dim} \operatorname{Im} \delta^{0}=4 k-2, \operatorname{dim}_{K} \mathrm{HH}^{0}(R)=k+s+2$.

Proof. It is easily seen that as a $K$-basis the vector space $\operatorname{Im} \delta^{0}$ admits the set that consists of the following elements:

$$
\begin{array}{ll}
\left(a^{i}+g^{i}, \mathrm{O}_{3}\right) & \text { for } 1 \leq i \leq k-1 \\
\left(\alpha g^{i}, \beta a^{i}, \mathrm{O}_{2}\right) & \text { for } 1 \leq i \leq k-1 \\
\left(\alpha g^{i}, 0, \gamma b^{i}, 0\right) & \text { for } 1 \leq i \leq k-1 \\
\left(0, \beta \alpha g^{i}, \alpha \gamma b^{i}, 0\right) & \text { for } 0 \leq i \leq k-1 \\
(0, \beta, \gamma, 0) . &
\end{array}
$$

Indeed, it suffices to consider the values of $\delta^{0}$ at the elements of the form $\left(r_{0}, 0\right)$ (respectively, of the form $\left(0, r_{1}\right)$ ), where $r_{0}$ runs through the set $\mathcal{B}_{00}$ (respectively, $r_{1}$ runs 
through the set $\left.\mathcal{B}_{11}\right)$. Then we prove that the set of elements indicated in (4.2) generates the vector space $\operatorname{Im} \delta^{0}$ and is linearly independent. Hence, we have $\operatorname{Im} \delta^{0}=4 k-2$ and

$$
\begin{aligned}
\operatorname{dim}_{K} \mathrm{HH}^{0}(R)=\operatorname{dim}_{K} \operatorname{Ker} \delta^{0} & =\operatorname{dim}_{K} \operatorname{Hom}_{\Lambda}\left(Q_{0}, R\right)-\operatorname{dim}_{K} \operatorname{Im} \delta^{0} \\
& =(5 k+s)-(4 k-2)=k+s+2 .
\end{aligned}
$$

Upon the identification mentioned above, the differential

$$
\delta^{1}: \operatorname{Hom}_{\Lambda}\left(Q_{1}, R\right) \rightarrow \operatorname{Hom}_{\Lambda}\left(Q_{2}, R\right)
$$

can be described as follows: for any $r_{i j} \in e_{i} R e_{j}(i, j \in\{0,1\})$ we have

$$
\delta^{1}\left(r_{00}, r_{10}, r_{01}, r_{11}\right)=\left(t_{00}, t_{11}, t_{00}^{\prime}, t_{10}, t_{11}^{\prime}, t_{01}\right),
$$

where

$$
\begin{aligned}
t_{00}= & \sum_{i=0}^{k-1} \gamma \beta a^{i} \cdot r_{00} \cdot g^{k-1-i}+\sum_{i=0}^{k-1} a^{i} \cdot r_{00} \cdot \gamma \beta a^{k-1-i}+\sum_{i=0}^{k-1} \alpha \gamma b^{i} \cdot r_{10} \cdot a^{k-1-i} \\
+ & \sum_{i=0}^{k-1} \gamma b^{i} \cdot r_{10} \cdot \alpha g^{k-1-i}+\sum_{i=0}^{k-1} g^{i} \cdot r_{01} \cdot \beta \alpha g^{k-1-i}+\sum_{i=0}^{k-1} \alpha g^{i} \cdot r_{01} \cdot \beta a^{k-1-i} \\
t_{11}=\sum_{i=0}^{k-1} \beta a^{i} \cdot r_{00} \cdot \gamma b^{k-1-i}+\sum_{i=0}^{k-1} b^{i} \cdot r_{10} \cdot \alpha \gamma b^{k-1-i} & +\sum_{i=0}^{k-1} \beta \alpha g^{i} \cdot r_{01} \cdot b^{k-1-i}+\sum_{i=0}^{s-1} \eta^{i} \cdot r_{11} \cdot \eta^{s-1-i} \\
t_{00}^{\prime}= & \alpha r_{00}+r_{00} \alpha+\sum_{i=0}^{k-1} \gamma \beta a^{i} \cdot r_{00} \cdot g^{k-1-i} \\
& +\sum_{i=0}^{k-1} \gamma b^{i} \cdot r_{10} \cdot \alpha g^{k-1-i}+\sum_{i=0}^{k-1} g^{i} \cdot r_{01} \cdot \beta \alpha g^{k-1-i}
\end{aligned}
$$

Proposition 4.4. (a) Suppose that $k$ and $s$ are even. Then the vector space $\operatorname{Im} \delta^{1}$ admits a K-basis formed by the following elements:

$$
\begin{array}{ll}
\left(\mathrm{O}_{2}, \alpha g^{i}, \mathrm{O}_{3}\right) & \text { for } 1 \leq i \leq k-1 ; \\
\left(\mathrm{O}_{2}, a^{i}+g^{i}, \mathrm{O}_{3}\right) & \text { for } 1 \leq i \leq k-1 ; \\
\left(\mathrm{O}_{4}, b^{i}, 0\right) & \text { for } 1 \leq i \leq k ; \\
\left(\mathrm{O}_{3}, \beta a^{i}, 0, \gamma b^{i}\right) & \text { for } 1 \leq i \leq k-1 ; \\
\left(\mathrm{O}_{2}, \gamma \beta a^{k-1}, \mathrm{O}_{3}\right), & \\
\left(\mathrm{O}_{3}, \beta, 0, \gamma\right) . &
\end{array}
$$

(b) Suppose that $k$ is even and $s$ is odd. In order to get a basis of the vector space $\operatorname{Im} \delta^{1}$, in the set described in part (a) we need to replace the element $\left(\mathrm{O}_{3}, \beta, 0, \gamma\right)$ in (4.3) by the element $\left(0, \eta^{s-1}, 0, \beta, 0, \gamma\right)$ and to adjoin the element $\left(0, \eta^{s}, \mathrm{O}_{4}\right)$ to this set.

(c) Suppose that $s$ is even and $k$ is odd. In order to get a basis of the vector space $\operatorname{Im} \delta^{1}$, we need to adjoin the element $\left(0, b^{k}, g^{k}, \mathrm{O}_{3}\right)$ to the set described in part (a).

(d) Finally, suppose that $k$ and $s$ are odd. In order to get a basis of the vector space $\operatorname{Im} \delta^{1}$, in the set described in part (a) we need to replace the element $\left(\mathrm{O}_{3}, \beta, 0, \gamma\right)$ in (4.3) by the element $\left(0, \eta^{s-1}, 0, \beta, 0, \gamma\right)$ and to adjoin the elements $\left(0, \eta^{s}, \mathrm{O}_{4}\right)$ and $\left(0, b^{k}, g^{k}, \mathrm{O}_{3}\right)$ to this set. 
Proof. As in the proof of Proposition 4.3, it suffices to consider the values of $\delta^{1}$ at the points of the form $\left(r_{00}, \mathrm{O}_{3}\right)$ (respectively, of the form $\left(0, r_{10}, \mathrm{O}_{2}\right)$, or $\left(\mathrm{O}_{2}, r_{01}, 0\right)$, or $\left.\left(\mathrm{O}_{3}, r_{11}\right)\right)$, where $r_{i j}$ runs through the subset $\mathcal{B}_{i j}(i, j \in\{0,1\})$ of the standard basis of the algebra $R$ (see (3.1)). Then we pick a basis of the vector space $\operatorname{Im} \delta^{1}$ from the resulting set of values of $\delta^{1}$, analyzing each case separately.

Corollary 4.5.
\[ \operatorname{dim}_{K} \operatorname{Im} \delta^{1}= \begin{cases}4 k+1 & \text { if } k \text { and } s \text { are odd, } \\ 4 k & \text { if exactly one number among } k \text { and } s \text { is even, } \\ 4 k-1 & \text { if } k \text { and } s \text { are even; }\end{cases} \]

$\operatorname{dim}_{K} \operatorname{Ker} \delta^{1}= \begin{cases}5 k+s-1 & \text { if } k \text { and } s \text { are odd, } \\ 5 k+s & \text { if exactly one number among } k \text { and } s \text { is even, } \\ 5 k+s+1 & \text { if } k \text { and } s \text { are even; }\end{cases}$

$\operatorname{dim}_{K} \operatorname{HH}^{1}(R)= \begin{cases}k+s+1 & \text { if } k \text { and } s \text { are odd, } \\ k+s+2 & \text { if exactly one number among } k \text { and } s \text { is even, } \\ k+s+3 & \text { if } k \text { and } s \text { are even. }\end{cases}$

Now we study the second differential

$$
\delta^{2}: \operatorname{Hom}_{\Lambda}\left(Q_{2}, R\right) \rightarrow \operatorname{Hom}_{\Lambda}\left(Q_{3}, R\right) .
$$

Under the above assumptions, it is described by the following formula: for any $r_{00}, r_{00}^{\prime} \in$ $e_{0} R e_{0}, r_{11}, r_{11}^{\prime} \in e_{1} R e_{1}, r_{10} \in e_{1} R e_{0}, r_{01} \in e_{0} R e_{1}$ we have

$$
\delta^{2}\left(r_{00}, r_{11}, r_{00}^{\prime}, r_{10}, r_{11}^{\prime}, r_{01}\right)=\left(t_{00}, t_{10}, t_{01}, t_{11}, t_{00}^{\prime}, t_{00}^{\prime \prime}, t_{11}^{\prime}, t_{11}^{\prime \prime}\right),
$$

where

$$
\begin{aligned}
& t_{00}=\alpha r_{00}+r_{00} \alpha+\gamma \beta a^{k-1} r_{00}+\gamma \beta a^{k-1} r_{00}^{\prime}+r_{00}^{\prime} \gamma \beta a^{k-1}, \\
& t_{10}=\beta r_{00}+r_{11} \beta+\eta^{s-1} r_{10}+r_{11}^{\prime} \beta \alpha g^{k-1}, \\
& t_{01}=r_{00} \gamma+\gamma r_{11}+\alpha \gamma b^{k-1} r_{11}^{\prime}+r_{01} \eta^{s-1}, \\
& t_{11}=\eta r_{11}+r_{11} \eta+r_{10} \alpha \gamma b^{k-1}+\beta \alpha g^{k-1} r_{01}, \\
& t_{00}^{\prime}=r_{00} \alpha+\alpha r_{00}^{\prime}+r_{00}^{\prime} \alpha, \quad t_{00}^{\prime \prime}=\gamma r_{10}+r_{01} \beta, \\
& t_{11}^{\prime}=r_{10} \gamma+\eta r_{11}^{\prime}, \quad t_{11}^{\prime \prime}=r_{11}^{\prime} \eta+\beta r_{01} .
\end{aligned}
$$

Proposition 4.6. For any $k$ and $s(k \geq 1, s \geq 2)$, the vector space $\operatorname{Im} \delta^{2}$ admits a $K$-basis formed by the following elements:

$$
\begin{array}{ll}
\left(\alpha g^{i}, \beta a^{i}, \mathrm{O}_{6}\right) & \text { for } 1 \leq i \leq k-1 ; \\
\left(\alpha g^{i}, 0, \gamma b^{i}, \mathrm{O}_{5}\right) & \text { for } 1 \leq i \leq k-1 ; \\
\left(a^{i}+g^{i}, \mathrm{O}_{3}, g^{i}, \mathrm{O}_{3}\right) & \text { for } 1 \leq i \leq k-1 ; \\
\left(0, \beta \alpha g^{i}, \alpha \gamma b^{i}, \mathrm{O}_{5}\right) & \text { for } 1 \leq i \leq k-1 ; \\
\left(\mathrm{O}_{5}, a^{i}, 0, b^{i}\right) & \text { for } 1 \leq i \leq k-1 ; \\
\left(\mathrm{O}_{5}, \gamma \beta a^{i}, \mathrm{O}_{2}\right) & \text { for } 1 \leq i \leq k-1 ; \\
\left(\mathrm{O}_{5}, g^{i}, b^{i}, 0\right) & \text { for } 1 \leq i \leq k ; \\
\left(\mathrm{O}_{4}, \alpha g^{i}, \mathrm{O}_{3}\right) & \text { for } 1 \leq i \leq k-1 ; \\
\left(\mathrm{O}_{4}, a^{i}+g^{i}, \mathrm{O}_{3}\right) & \text { for } 1 \leq i \leq k-1 ; \\
\left(\mathrm{O}_{6}, \eta^{i}, \eta^{i}\right) & \text { for } 1 \leq i \leq s ;
\end{array}
$$




$$
\begin{aligned}
& \left(a^{k}, \beta \alpha, \alpha \gamma, \mathrm{O}_{5}\right), \quad\left(\gamma \beta a^{k-1}, \mathrm{O}_{3}, \alpha, \mathrm{O}_{3}\right), \quad\left(\mathrm{O}_{4}, a^{k}, \mathrm{O}_{3}\right), \\
& \left(0, \beta, \gamma, \mathrm{O}_{5}\right), \quad\left(\mathrm{O}_{3}, b^{k}, 0, \gamma \beta, \mathrm{O}_{2}\right) .
\end{aligned}
$$

Proof. The proof is similar to that of Proposition 4.4.

\section{Corollary 4.7.}

$$
\begin{array}{r}
\operatorname{dim}_{K} \operatorname{Im} \delta^{2}=9 k+s-3 ; \\
\operatorname{dim}_{K} \operatorname{Ker} \delta^{2}=5 k+s+3 ;
\end{array}
$$$$
\operatorname{dim}_{K} \mathrm{HH}^{2}(R)= \begin{cases}k+s+4, & \text { if } k \text { and } s \text { are even, } \\ k+s+3, & \text { if exactly one number among } k \text { and } s \text { is even, } \\ k+s+2, & \text { if } k \text { and } s \text { are odd. }\end{cases}
$$

The differential

$$
\delta^{3}: \operatorname{Hom}_{\Lambda}\left(Q_{3}, R\right) \rightarrow \operatorname{Hom}_{\Lambda}\left(Q_{4}, R\right)
$$

is described as follows: for any $r_{00}, r_{00}^{\prime}, r_{00}^{\prime \prime} \in e_{0} R e_{0}, r_{11}, r_{11}^{\prime}, r_{11}^{\prime \prime} \in e_{1} R e_{1}, r_{10} \in e_{1} R e_{0}$, $r_{01} \in e_{0} R e_{1}$, we have

$$
\delta^{3}\left(r_{00}, r_{10}, r_{01}, r_{11}, r_{00}^{\prime}, r_{00}^{\prime \prime}, r_{11}^{\prime}, r_{11}^{\prime \prime}\right)=\left(t_{00}, t_{11}, t_{00}^{\prime}, t_{10}, t_{11}^{\prime}, t_{01}, t_{00}^{\prime \prime}, t_{10}^{\prime}, t_{01}^{\prime}, t_{11}^{\prime \prime}\right),
$$

where

$$
\begin{aligned}
t_{00} & =\sum_{i=0}^{k-1} \gamma \beta a^{i} \cdot r_{00} \cdot g^{k-1-i}+\sum_{i=0}^{k-1} a^{i} \cdot r_{00} \cdot \gamma \beta a^{k-1-i} \\
& +\sum_{i=0}^{k-1} \alpha \gamma b^{i} \cdot r_{10} \cdot a^{k-1-i}+\sum_{i=0}^{k-1} \gamma b^{i} \cdot r_{10} \cdot \alpha g^{k-1-i} \\
& +\sum_{i=0}^{k-1} g^{i} \cdot r_{01} \cdot \beta \alpha g^{k-1-i}+\sum_{i=0}^{k-1} \alpha g^{i} \cdot r_{01} \cdot \beta a^{k-1-i}+\gamma b^{k-1} \cdot r_{10} \cdot \gamma \beta a^{k-1}, \\
t_{11}= & \sum_{i=0}^{k-1} \beta a^{i} \cdot r_{00} \cdot \gamma b^{k-1-i}+\sum_{i=0}^{k-1} b^{i} \cdot r_{10} \cdot \alpha \gamma b^{k-1-i} \\
& +\sum_{i=0}^{k-1} \beta \alpha g^{i} \cdot r_{01} \cdot b^{k-1-i}+\sum_{i=0}^{s-1} \eta^{i} \cdot r_{11} \cdot \eta^{s-1-i}, \\
t_{00}^{\prime}= & r_{00} \alpha+\alpha r_{00}+\gamma \beta a^{k-1} r_{00}^{\prime}+r_{00}^{\prime} \gamma \beta a^{k-1}, \\
t_{10}= & \eta r_{10}+r_{11} \beta+\beta \alpha g^{k-1} r_{00}^{\prime \prime}+r_{11}^{\prime} \beta \alpha g^{k-1}, \\
t_{11}^{\prime}= & r_{10} \gamma+\beta r_{01}+\eta^{s-1} r_{11}^{\prime}+r_{11}^{\prime \prime} \eta^{s-1}, \\
t_{01}= & r_{01} \eta+\gamma r_{11}+r_{00}^{\prime \prime} \alpha \gamma b^{k-1}+\alpha \gamma b^{k-1} r_{11}^{\prime \prime}, \\
t_{00}^{\prime \prime}= & r_{00} \alpha+\alpha r_{00}^{\prime}+r_{00}^{\prime} \alpha+\gamma \beta a^{k-1} r_{00}^{\prime}, \\
t_{10}^{\prime}= & \beta r_{00}^{\prime \prime}+r_{11}^{\prime \prime} \beta, \quad t_{10}^{\prime}=r_{00}^{\prime \prime} \gamma+\gamma r_{11}^{\prime}, \quad t_{11}^{\prime \prime}=r_{11}^{\prime} \eta+\eta r_{11}^{\prime \prime} .
\end{aligned}
$$

Now, arguing as above, we can find a basis of the vector space $\operatorname{Im} \delta^{3}$.

Proposition 4.8. (a) Suppose that $k$ and $s$ are even. Then the vector space $\operatorname{Im} \delta^{3}$ admits a K-basis formed by the following elements:

$$
\begin{array}{ll}
\left(\mathrm{O}_{2}, \alpha g^{i}, \mathrm{O}_{7}\right) & \text { for } 1 \leq i \leq k-1 \\
\left(\mathrm{O}_{2}, a^{i}+g^{i}, \mathrm{O}_{3}, g^{i}, \mathrm{O}_{3}\right) & \text { for } 1 \leq i \leq k-1 \\
\left(\mathrm{O}_{3}, \beta a^{i}, 0, \gamma b^{i}, \mathrm{O}_{4}\right) & \text { for } 1 \leq i \leq k-1
\end{array}
$$




$$
\begin{array}{lll} 
& \left(\mathrm{O}_{4}, b^{i}, \mathrm{O}_{5}\right) & \text { for } 1 \leq i \leq k ; \\
& \left(\mathrm{O}_{6}, \alpha g^{i}, \mathrm{O}_{3}\right) & \text { for } 0 \leq i \leq k-1 ; \\
& \left(\mathrm{O}_{6}, a^{i}+g^{i}, \mathrm{O}_{3}\right) & \text { for } 1 \leq i \leq k-1 ; \\
\left(\mathrm{O}_{7}, \beta a^{i}, \mathrm{O}_{2}\right) & \text { for } 1 \leq i \leq k-1 ; \\
\left(\mathrm{O}_{7}, \beta \alpha g^{i}, \alpha \gamma b^{i}, 0\right) & \text { for } 0 \leq i \leq k-1 ; \\
\left(\mathrm{O}_{8}, \gamma b^{i}, 0\right) & \text { for } 1 \leq i \leq k-1 ; \\
& \left(\mathrm{O}_{9}, \eta^{i}\right) & \\
& \left(\mathrm{O}_{6}, \gamma \beta a^{k-1}, \mathrm{O}_{3}\right), \quad\left(\mathrm{O}_{3}, \beta \alpha g^{k-1}, \eta^{s-1}, \mathrm{O}_{3}, \gamma, \eta\right), & \\
& \left(\mathrm{O}_{6}, a^{k}, \mathrm{O}_{3}\right), \quad\left(\mathrm{O}_{3}, \beta \alpha g^{k-1}, 0, \alpha \gamma b^{k-1}, 0, \beta, \gamma, 0\right), & \\
& \left(\mathrm{O}_{3}, \beta, 0, \gamma, \mathrm{O}_{4}\right) . &
\end{array}
$$

(b) Suppose that $k$ is even and $s$ is odd. In order to get a basis of the vector space $\operatorname{Im} \delta^{3}$, in the set described in part (a) we need to replace the element $\left(\mathrm{O}_{3}, \beta, 0, \gamma, \mathrm{O}_{4}\right)$ in (4.4) by the element $\left(0, \eta^{s-1}, 0, \beta, 0, \gamma, \mathrm{O}_{4}\right)$ and to adjoin the element $\left(0, \eta^{s}, \mathrm{O}_{8}\right)$ to this set.

(c) Suppose that $s$ is even and $k$ is odd. In order to get a basis of the vector space $\operatorname{Im} \delta^{3}$, we need to adjoin the element $\left(0, \eta^{s}, \mathrm{O}_{8}\right)$ to the set described in part (a).

(d) Finally, suppose that $k$ and $s$ are odd. In order to get a basis of the vector space $\operatorname{Im} \delta^{3}$, we can take the set described in part (b).

\section{Corollary 4.9.}

$$
\begin{aligned}
\operatorname{dim}_{K} \operatorname{Im} \delta^{3} & = \begin{cases}9 k+s-2 & \text { if } k \text { and } s \text { are even } \\
9 k+s-1 & \text { otherwise }\end{cases} \\
\operatorname{dim}_{K} \operatorname{Ker} \delta^{3} & = \begin{cases}10 k+2 s+2 & \text { if } k \text { and } s \text { are even } \\
10 k+2 s+1 & \text { otherwise }\end{cases} \\
\operatorname{dim}_{K} \mathrm{HH}^{3}(R) & = \begin{cases}k+s+5 & \text { if } k \text { and } \text { s are even } \\
k+s+4 & \text { otherwise }\end{cases}
\end{aligned}
$$

Proof. The statement on $\operatorname{dim}_{K} \mathrm{HH}^{3}(R)$ follows from the description of the dimension of Ker $\delta^{3}$ and from Corollary 4.7 .

By similar arguments, we obtain the description of the kernel of the differential

$$
\delta^{4}: \operatorname{Hom}_{\Lambda}\left(Q_{4}, R\right) \rightarrow \operatorname{Hom}_{\Lambda}\left(Q_{5}, R\right) .
$$

Using the the form of the differential $d_{4}^{Q}$ in the bimodule resolution constructed in $\oint 3$, we deduce the following statement; the details of the corresponding calculation are left to the reader.

Proposition 4.10. (a) Suppose that $k \geq 2$. Then the vector space $\operatorname{Im} \delta^{4}$ admits a $K$-basis formed by the following elements:

$$
\begin{array}{ll}
\left(g^{i}+a^{i}, \mathrm{O}_{11}\right) & \text { for } 1 \leq i \leq k-1 ; \\
\left(\alpha g^{i}, 0, \gamma b^{i}, \mathrm{O}_{9}\right) & \text { for } 1 \leq i \leq k-1 ; \\
\left(0, \beta a^{i}, \gamma b^{i}, \mathrm{O}_{9}\right) & \text { for } 0 \leq i \leq k-1 ; \\
\left(0, \beta \alpha g^{i}, \alpha \gamma b^{i}, \mathrm{O}_{9}\right) & \text { for } 0 \leq i \leq k-1 ; \\
\left(\mathrm{O}_{4}, \alpha g^{i}, \mathrm{O}_{7}\right) & \text { for } 1 \leq i \leq k-1 ;
\end{array}
$$




$$
\begin{array}{ll}
\left(\mathrm{O}_{4}, a^{i}+g^{i}, \mathrm{O}_{3}, g^{i}, \mathrm{O}_{3}\right) & \text { for } 1 \leq i \leq k-1 ; \\
\left(\mathrm{O}_{5}, \gamma \beta a^{i}, \mathrm{O}_{6}\right) & \text { for } 1 \leq i \leq k-1 ; \\
\left(\mathrm{O}_{5}, g^{i}, b^{i}, \mathrm{O}_{5}\right) & \text { for } 1 \leq i \leq k ; \\
\left(\mathrm{O}_{5}, a^{i}, 0, b^{i}, \mathrm{O}_{4}\right) & \text { for } 1 \leq i \leq k-1 ; \\
\left(\mathrm{O}_{6}, \eta^{i}, \eta^{i}, \mathrm{O}_{4}\right) & \text { for } 2 \leq i \leq s ; \\
\left(\mathrm{O}_{8}, \alpha g^{i}, \mathrm{O}_{3}\right) & \text { for } 1 \leq i \leq k-1 ; \\
\left(\mathrm{O}_{8}, a^{i}+g^{i}, \mathrm{O}_{3}\right) & \text { for } 1 \leq i \leq k-1 ; \\
\left(\mathrm{O}_{9}, \beta a^{i}, 0, \gamma b^{i}\right) & \text { for } 0 \leq i \leq k-1 ; \\
\left(\mathrm{O}_{10}, b^{i}, 0\right) & \text { for } 1 \leq i \leq k ; \\
\left(\mathrm{O}_{3}, b^{k}, 0, \gamma \beta, \mathrm{O}_{6}\right),\left(\mathrm{O}_{4}, g^{k}, \mathrm{O}_{7}\right), & \\
\left(\mathrm{O}_{4}, \gamma \beta a^{k-1}, \mathrm{O}_{3}, \alpha, \mathrm{O}_{3}\right),\left(\mathrm{O}_{8}, g^{k}, \mathrm{O}_{3}\right), & \\
\left(\mathrm{O}_{6}, \eta, \eta, \mathrm{O}_{4}\right) . &
\end{array}
$$

(b) If $k=1$, then, in order to get a basis of the vector space $\operatorname{Im} \delta^{4}$, to the set of elements of the form (4.5)-(4.12) we need to adjoin the element

$$
\left(g, \mathrm{O}_{5}, \eta, \eta, \mathrm{O}_{4}\right) \text {. }
$$

Corollary 4.11. Suppose that $k, s \in \mathbb{N}, s \geq 2$. Then

$$
\begin{aligned}
\operatorname{dim} \operatorname{Im} \delta^{4} & =13 k+s-4 ; \\
\operatorname{dim}_{K} \operatorname{Ker} \delta^{4} & =10 k+2 s+4 ; \\
\operatorname{dim}_{K} \operatorname{HH}^{4}(R) & = \begin{cases}k+s+6 & \text { if } k \text { and } s \text { are even, } \\
k+s+5 & \text { otherwise. }\end{cases}
\end{aligned}
$$

Put $\mathcal{X}^{\bullet}:=\operatorname{Hom}_{\Lambda}\left(X_{\bullet}, R\right)$, where $X_{\bullet}$ is the complex occurring in Proposition 3.3. As above, we identify the elements $f \in \operatorname{Hom}_{\Lambda}\left(X_{n}, R\right) \subset \operatorname{Hom}_{\Lambda}\left(Q_{n}, R\right)$ with the corresponding sets of the values $f\left(e_{i} \otimes e_{j}\right)$ (cf. Remark 4.1).

The description of the bimodule resolution of $R$ constructed in $\$ 3$ shows that the complex $\mathcal{X}^{\bullet}$ is 6 -periodic in large degrees, more precisely, for any $n \geq 9$ we have

$$
\delta_{\mathcal{X} \bullet}^{n}=\delta_{\mathcal{X} \bullet}^{n-6}
$$

whence

$$
\mathrm{H}^{n}\left(\mathcal{X}^{\bullet}\right) \simeq \mathrm{H}^{n-6}\left(\mathcal{X}^{\bullet}\right)
$$

for all $n \geq 10$.

Remark 4.12. In fact, the complex $X_{\bullet}$ decomposes into the direct sum of subcomplexes $X_{\bullet}=Y_{\bullet} \oplus Z_{\bullet}$, where $Y_{n}=P_{00}^{2}$ corresponds to the direct summands at the first and fifth places in the standard decomposition of the module $X_{n}$ (see Remark 4.1), and $Z_{n}$ corresponds to the remaining direct summands in that decomposition. Then we obtain the decomposition $\mathcal{X}^{\bullet}=\mathcal{Y}^{\bullet} \oplus \mathcal{Z}^{\bullet}$ with $\mathcal{Y}^{\bullet}=\operatorname{Hom}_{\Lambda}\left(Y_{\bullet}, R\right)$ and $\mathcal{Z}^{\bullet}=\operatorname{Hom}_{\Lambda}\left(Z_{\bullet}, R\right)$. Moreover, the subcomplex $\mathcal{Y}_{\bullet}$ is 2-periodic (starting with degree 2), and $\mathcal{Z}_{\bullet}$ is 3-periodic (starting with degree 3 ).

Proposition 4.13. For any $n \geq 4$, we have

$$
\operatorname{dim}_{K} \mathrm{H}^{n}\left(\mathcal{X}^{\bullet}\right)= \begin{cases}4 & \text { if } n \equiv 1 \quad(\bmod 3), \\ 5 & \text { otherwise } .\end{cases}
$$


Proof. Using Remark 4.12, we see that it suffices to calculate cohomology $\mathrm{H}^{n}\left(\mathcal{Y}^{\bullet}\right)$ and $\mathrm{H}^{n}\left(\mathcal{Z}^{\bullet}\right)$ of the corresponding subcomplexes.

(a) It is easy to check that, for even $n(n \geq 4)$, as a basis of the vector space $\operatorname{Ker} \delta_{\mathcal{Y}}^{n}$. we can take the set consisting of the elements

$$
\begin{array}{ll}
\left(\alpha g^{i}, \mathrm{O}_{7}\right) & \text { for } 1 \leq i \leq k-1 ; \\
\left(g^{i}+a^{i}, \mathrm{O}_{3}, g^{i}, \mathrm{O}_{3}\right) & \text { for } 1 \leq i \leq k-1 ; \\
\left(\mathrm{O}_{4}, \alpha g^{i}, \mathrm{O}_{3}\right) & \text { for } 0 \leq i \leq k-1 ; \\
\left(\mathrm{O}_{4}, g^{i}+a^{i}, \mathrm{O}_{3}\right) & \text { for } 1 \leq i \leq k-1 ; \\
\left(a^{k}, \mathrm{O}_{7}\right), \quad\left(\mathrm{O}_{4}, e_{0}, \mathrm{O}_{3}\right), & \\
\left(\mathrm{O}_{4}, \gamma \beta a^{k-1}, \mathrm{O}_{3}\right), \quad\left(\mathrm{O}_{4}, a^{k}, \mathrm{O}_{3}\right) . &
\end{array}
$$

(b) If $n$ is odd $(n>4)$, then in order to get a basis of the vector space Ker $\delta_{\mathcal{Y} \bullet}^{n}$, in the set indicated in (4.14) we need to replace the elements $\left(\mathrm{O}_{4}, \alpha, \mathrm{O}_{3}\right)$ and $\left(\mathrm{O}_{4}, e_{0}, \mathrm{O}_{3}\right)$ by the elements $\left(\alpha, \mathrm{O}_{3}, \alpha, \mathrm{O}_{3}\right)$ and $\left(\alpha+\gamma \beta a^{k-1}, \mathrm{O}_{7}\right)$.

(c) Accordingly, in order to get a basis of the vector space $\operatorname{Im} \delta_{\mathcal{Y}}^{n}$ for even $n(n \geq 4)$ it suffices to remove the elements

$$
\left(\alpha, \mathrm{O}_{3}, \alpha, \mathrm{O}_{3}\right), \quad\left(\mathrm{O}_{4}, \gamma \beta a^{k-1}, \mathrm{O}_{3}\right)
$$

from the basis of the vector space $\operatorname{Ker} \delta_{\mathcal{Y}}^{n+1}$ (see part (b) of the proof).

If $n$ is odd $(n>4)$, then in order to get a basis of the vector space $\operatorname{Im} \delta_{\mathcal{Y}}^{n}$. it suffices to remove the elements

$$
\left(a^{k}, \mathrm{O}_{7}\right), \quad\left(\mathrm{O}_{4}, e_{0}, \mathrm{O}_{3}\right)
$$

from the basis of the vector space $\operatorname{Ker} \delta_{\mathcal{Y}}^{n+1}$ (see part (a) of the proof).

Consequently, for any $n \geq 4$ we have

$$
\operatorname{dim} \operatorname{Ker} \delta_{\mathcal{Y} \bullet}^{n}=4 k+1, \quad \operatorname{dim} \operatorname{Im} \delta_{\mathcal{Y} \bullet}^{n}=4 k-1,
$$

whence

$$
\operatorname{dim} H^{n}\left(\mathcal{Y}^{\bullet}\right)=2 .
$$

(d) Now we calculate $\mathrm{H}^{n}\left(\mathcal{Z}^{\bullet}\right)$; first, we describe $\operatorname{Ker} \delta_{\mathcal{Z}}^{n} \bullet$ If $n \equiv 0(\bmod 3)(n>3)$, then as a basis of the vector space $\operatorname{Ker} \delta_{\mathcal{Y}}^{n}$. we can take the set consisting of the elements

$$
\begin{array}{ll}
\left(0, \beta \alpha g^{i}, \alpha \gamma b^{i}, \mathrm{O}_{5}\right) & \text { for } 0 \leq i \leq k-1 ; \\
\left(0, \beta a^{i}, \mathrm{O}_{6}\right) & \text { for } 0 \leq i \leq k-1 ; \\
\left(\mathrm{O}_{2}, \gamma b^{i}, \mathrm{O}_{5}\right) & \text { for } 0 \leq i \leq k-1 ; \\
\left(\mathrm{O}_{3}, \eta^{i}, \mathrm{O}_{4}\right) & \text { for } 1 \leq i \leq s ; \\
\left(\mathrm{O}_{5}, a^{i}, 0, b^{i}\right) & \text { for } 1 \leq i \leq k-1 ; \\
\left(\mathrm{O}_{5}, g^{i},, b^{i}, 0\right) & \text { for } 1 \leq i \leq k-1 ; \\
\left(\mathrm{O}_{5}, \gamma \beta a^{i}, \mathrm{O}_{2}\right) & \text { for } 0 \leq i \leq k-1 ; \\
\left(\mathrm{O}_{6}, \eta^{i}, \eta^{i}\right) & \text { for } 1 \leq i \leq s-1 ; \\
\left(\mathrm{O}_{5}, e_{0}, e_{1}, e_{1}\right), \quad\left(\mathrm{O}_{5}, a^{k}, \mathrm{O}_{2}\right), & \\
\left(\mathrm{O}_{6}, \eta^{s}, 0\right), \quad\left(\mathrm{O}_{7}, \eta^{s}\right) . &
\end{array}
$$


If $n \equiv 1(\bmod 3)(n \geq 4)$, then as a basis of the vector space $\operatorname{Ker} \delta_{\mathcal{Z}}^{n} \bullet$ we can take the set consisting of the elements

$$
\begin{array}{ll}
\left(0, \beta a^{i}, 0, \gamma b^{i}, \mathrm{O}_{4}\right) & \text { for } 0 \leq i \leq k-1 ; \\
\left(\mathrm{O}_{2}, b^{i}, \mathrm{O}_{5}\right) & \text { for } 1 \leq i \leq k ; \\
\left(\mathrm{O}_{5}, \beta \alpha g^{i}, \alpha \gamma b^{i}, 0\right) & \text { for } 0 \leq i \leq k-1 ; \\
\left(\mathrm{O}_{5}, \beta a^{i}, \mathrm{O}_{2}\right) & \text { for } 1 \leq i \leq k-1 ; \\
\left(\mathrm{O}_{6}, \gamma b^{i}, 0\right) & \text { for } 1 \leq i \leq k-1 ; \\
\left(\mathrm{O}_{7}, \eta^{i}\right) & \text { for } 2 \leq i \leq s ; \\
\left(0, \beta \alpha g^{k-1}, \eta^{s-1}, \alpha \gamma b^{k-1}, \mathrm{O}_{4}\right),\left(0, \beta \alpha g^{k-1}, \mathrm{O}_{4}, \gamma, 0\right), & \\
\left(\mathrm{O}_{3}, \alpha \gamma b^{k-1}, 0, \beta, \mathrm{O}_{2}\right), & \left(\mathrm{O}_{2}, \eta^{s-1}, \mathrm{O}_{4}, \eta\right) .
\end{array}
$$

Next, if $n \equiv 2(\bmod 3)(n>4)$, then as a basis of the vector space $\operatorname{Ker} \delta_{\mathcal{Z}}^{n}$ we can take the set consisting of the elements

$$
\begin{array}{lr}
\left(0, a^{i}, 0, b^{i}, \mathrm{O}_{4}\right) & \text { for } 1 \leq i \leq k-1 ; \\
\left(0, g^{i}, b^{i}, \mathrm{O}_{5}\right) & \text { for } 1 \leq i \leq k-1 ; \\
\left(0, \gamma \beta a^{i}, \mathrm{O}_{6}\right) & \text { for } 0 \leq i \leq k-1 ; \\
\left(\mathrm{O}_{2}, \eta^{i}, \eta^{i}, \mathrm{O}_{4}\right) & \text { for } 1 \leq i \leq s-1 ; \\
\left(\mathrm{O}_{5}, \beta a^{i}, 0, \gamma b^{i}\right) & \text { for } 0 \leq i \leq k-1 ; \\
\left(\mathrm{O}_{6}, b^{i}, 0\right) & \text { for } 1 \leq i \leq k-1 ; \\
\left(0, e_{0}, e_{1}, e_{1}, \mathrm{O}_{4}\right),\left(0, a^{k}, \mathrm{O}_{6}\right),\left(\mathrm{O}_{2}, \eta^{s}, \mathrm{O}_{5}\right) & \\
\left(\mathrm{O}_{3}, \eta^{s}, \mathrm{O}_{4}\right),\left(\mathrm{O}_{5}, \beta \alpha g^{k-1}, \eta^{s-1}, \alpha \gamma b^{k-1}\right),\left(\mathrm{O}_{6}, \eta^{s}, 0\right) .
\end{array}
$$

(e) Finally, we describe $\operatorname{Im} \delta_{\mathcal{Z}}^{n} \bullet$ If $n \equiv 0(\bmod 3)(n>3)$, in order to get a basis of the vector space $\operatorname{Im} \delta_{\mathcal{Z}}^{n}$, in the basis of the vector space $\operatorname{Ker} \delta_{\mathcal{Z}}^{n+1}$ (here we use (4.17), since $n+1 \equiv 1(\bmod 3))$ we need to replace the element $\left(0, \beta \alpha g^{k-1}, \mathrm{O}_{4}, \gamma, 0\right)$ by the element $\left(0, \beta \alpha g^{k-1}, 0, \alpha \gamma b^{k-1}, 0, \beta, \gamma, 0\right)$, the element $\left(\mathrm{O}_{2}, \eta^{s-1}, \mathrm{O}_{4}, \eta\right)$ by the element $\left(0, \beta \alpha g^{k-1}, \eta^{s-1}, \mathrm{O}_{3}, \gamma, \eta\right)$, and to remove the elements

$$
\left(0, \beta \alpha g^{k-1}, \eta^{s-1}, \alpha \gamma b^{k-1}, \mathrm{O}_{4}\right), \quad\left(\mathrm{O}_{3}, \alpha \gamma b^{k-1}, 0, \beta, \mathrm{O}_{2}\right) .
$$

If $n \equiv 1(\bmod 3)(n \geq 4)$, then, in order to get a basis of the vector space $\operatorname{Im} \delta_{\mathcal{Z}}^{n} \bullet$, in the basis of the vector space $\operatorname{Ker} \delta_{\mathcal{Z}}^{n+1}$ (see (4.18) ) we need to replace the element $\left(\mathrm{O}_{2}, \eta^{s}, \mathrm{O}_{5}\right)$ by $\left(0, a^{k}, \eta^{s}, \mathrm{O}_{5}\right)$, to replace $\left(\mathrm{O}_{3}, \eta^{s}, \mathrm{O}_{4}\right)$ by $\left(\mathrm{O}_{2}, \eta^{s}, \eta^{s}, \mathrm{O}_{4}\right)$, and to remove the elements

$$
\left(0, e_{0}, e_{1}, e_{1}, \mathrm{O}_{4}\right), \quad\left(0, a^{k}, \mathrm{O}_{6}\right), \quad\left(\mathrm{O}_{5}, \beta \alpha g^{k-1}, \eta^{s-1}, \alpha \gamma b^{k-1}\right) .
$$

If $n \equiv 2(\bmod 3)(n>4)$, then, in order to get a basis of the vector space $\operatorname{Im} \delta_{\mathcal{Z}}^{n}$, in the basis of the vector space $\operatorname{Ker} \delta_{\mathcal{Z}}^{n+1}$ (see (4.16) ) we need to replace the elements

$$
\left(\mathrm{O}_{2}, \gamma, \mathrm{O}_{5}\right), \quad\left(\mathrm{O}_{3}, \eta, \mathrm{O}_{4}\right), \quad\left(\mathrm{O}_{6}, \eta^{s}, 0\right), \quad\left(\mathrm{O}_{7}, \eta^{s}\right)
$$

by the elements

$$
\left(0, \beta, \gamma, \mathrm{O}_{5}\right), \quad\left(\mathrm{O}_{2}, \gamma, \eta, \mathrm{O}_{4}\right), \quad\left(\mathrm{O}_{5}, a^{k}, \eta^{s}, 0\right), \quad\left(\mathrm{O}_{6}, \eta^{s}, \eta^{s}\right),
$$

respectively, and to remove the elements

$$
\left(0, \beta, \mathrm{O}_{6}\right), \quad\left(\mathrm{O}_{5}, e_{0}, e_{1}, e_{1}\right), \quad\left(\mathrm{O}_{5}, a^{k}, \mathrm{O}_{2}\right) .
$$


The above calculation shows that, for any $n \geq 4$,

$$
\operatorname{dim} H^{n}\left(\mathcal{Z}^{\bullet}\right)= \begin{cases}2 & \text { if } n \equiv 1 \quad(\bmod 3), \\ 3 & \text { otherwise }\end{cases}
$$

and it suffices to use (4.15) to get the desired statement.

From the proof of Proposition 4.13 we obtain the following description of the basis of cohomologies $\mathrm{H}^{n}\left(\mathcal{Y}^{\bullet}\right)$ and $\mathrm{H}^{n}\left(\mathcal{Z}^{\bullet}\right)$.

Corollary 4.14. Suppose that $n \geq 4$.

1) If $n$ is even, then

$$
\mathrm{H}^{n}\left(\mathcal{Y}^{\bullet}\right)={ }_{K}\left\langle\left(a^{k}, \mathrm{O}_{7}\right),\left(\mathrm{O}_{4}, e_{0}, \mathrm{O}_{3}\right)\right\rangle
$$

if $n$ is odd, then

$$
\mathrm{H}^{n}\left(\mathcal{Y}^{\bullet}\right)={ }_{K}\left\langle\left(\alpha, \mathrm{O}_{3}, \alpha, \mathrm{O}_{3}\right),\left(\mathrm{O}_{4}, \gamma \beta a^{k-1}, \mathrm{O}_{3}\right)\right\rangle .
$$

2) If $n \equiv 0(\bmod 3)$, then

$$
\mathrm{H}^{n}\left(\mathcal{Z}^{\bullet}\right)={ }_{K}\left\langle\left(0, \beta, \mathrm{O}_{6}\right),\left(\mathrm{O}_{5}, e_{0}, e_{1}, e_{1}\right),\left(\mathrm{O}_{5}, a^{k}, \mathrm{O}_{2}\right)\right\rangle ;
$$

if $n \equiv 1(\bmod 3)$, then

$$
\mathrm{H}^{n}\left(\mathcal{Z}^{\bullet}\right)={ }_{K}\left\langle\left(0, \beta \alpha g^{k-1}, \eta^{s-1}, \alpha \gamma b^{k-1}, \mathrm{O}_{4}\right), \quad\left(\mathrm{O}_{3}, \alpha \gamma b^{k-1}, 0, \beta, \mathrm{O}_{2}\right)\right\rangle ;
$$

and if $n \equiv 2(\bmod 3)$, then

$$
\mathrm{H}^{n}\left(\mathcal{Z}^{\bullet}\right)={ }_{K}\left\langle\left(0, e_{0}, e_{1}, e_{1}, \mathrm{O}_{4}\right),\left(0, a^{k}, \mathrm{O}_{6}\right), \quad\left(\mathrm{O}_{5}, \beta \alpha g^{k-1}, \eta^{s-1}, \alpha \gamma b^{k-1}\right)\right\rangle .
$$

Proposition 4.15. For any $n \geq 5$, we have

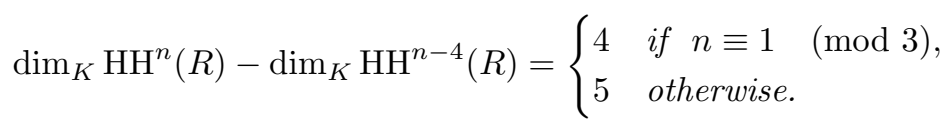

Proof. Applying the functor $\operatorname{Hom}_{\Lambda}(-, R)$ to the short exact sequence (3.3), we obtain a short exact sequence of complexes

$$
0 \rightarrow \operatorname{Hom}_{\Lambda}(Q \bullet[-4], R) \stackrel{\pi^{*}}{\rightarrow} \operatorname{Hom}_{\Lambda}(Q \bullet, R) \stackrel{\imath^{*}}{\rightarrow} \mathcal{X}^{\bullet} \rightarrow 0
$$

(where $\mathcal{X}^{\bullet}=\operatorname{Hom}_{\Lambda}\left(X_{\bullet}, R\right)$ ), which, in its turn, gives a long exact cohomology sequence

$$
\ldots \stackrel{\Delta^{n-1}}{\longrightarrow} \mathrm{HH}^{n-4}(R) \stackrel{\pi^{*}}{\longrightarrow} \mathrm{HH}^{n}(R) \stackrel{\imath^{*}}{\rightarrow} \mathrm{H}^{n}\left(\mathcal{X} \bullet \stackrel{\Delta^{n}}{\longrightarrow} \mathrm{HH}^{n-3}(R) \stackrel{\pi^{*}}{\longrightarrow} \ldots\right.
$$

Lemma 4.16. For any $n \geq 4$, we have $\Delta^{n}=0$.

Proof. We recall that the connecting homomorphism $\Delta^{n}$ is constructed as follows. Let $f \in \operatorname{Ker} \delta_{\mathcal{X}}^{n}$, and put $\tilde{f}:=(\mathrm{O}, f) \in \operatorname{Hom}_{\Lambda}\left(Q_{n}, R\right)$, i.e., $\tilde{f}$ is the extension of $f$ by zero to the entire module $Q_{n}=Q_{n-4} \oplus X_{n}$. Since $\imath^{*}(\tilde{f})=f$, there exists $g \in \operatorname{Hom}_{\Lambda}\left(Q_{n-3}, R\right)$ such that $\pi^{*}(g)=\delta^{n}(\tilde{f})$, where $g$ is a cocycle, and then we define $\Delta(\operatorname{cl} f):=\operatorname{cl} g$.

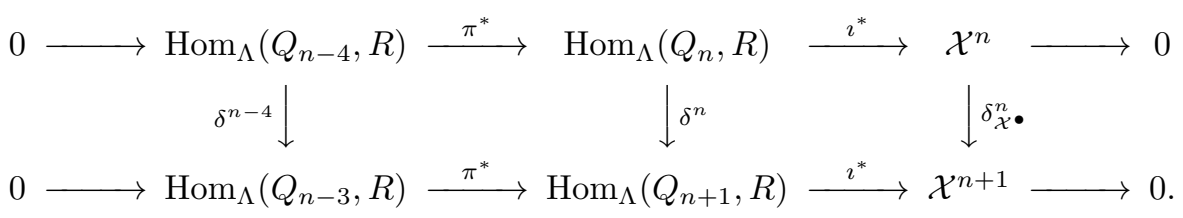

1) Suppose that $n \equiv 1(\bmod 6)(n \geq 4)$ and put $f=\left(s_{1}, \ldots, s_{8}\right) \in \operatorname{Ker} \delta_{\mathcal{X}}^{n}$; here the $s_{t}, 1 \leq t \leq 8$, lie in the suitable $P_{i j}$. We introduce the abbreviated notation $S_{f}:=$ 
$\left(s_{1}, s_{2}, s_{3}, s_{4}\right)$. By Corollary 4.14, we may assume that $f$ is one of the elements in (4.20), (4.22). We observe that the differential $d_{n}^{Q} \bullet$ has the following "block-triangular" form:

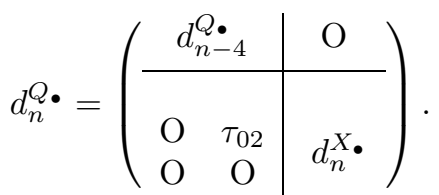

Then, in the previous notation, we have

$$
\delta^{n}(\tilde{f})=\left(\mathrm{O}, \tau_{02}^{*}\left(S_{f}\right), \mathrm{O}_{8}\right) .
$$

If $f_{1}=\left(\alpha, \mathrm{O}_{3}, \alpha, \mathrm{O}_{3}\right)$, then, clearly, $\tau_{02}^{*}\left(S_{f_{1}}\right)=0$, whence $\Delta^{n}\left(\operatorname{cl} f_{1}\right)=0$. For $f_{2}=$ $\left(0, \beta \alpha g^{k-1}, \eta^{s-1}, \alpha \gamma b^{k-1}, \mathrm{O}_{4}\right)$ and $f_{3}=\left(\mathrm{O}_{3}, \alpha \gamma b^{k-1}, 0, \beta, \mathrm{O}_{2}\right)$, again we have $\tau_{02}^{*}\left(S_{f_{2}}\right)=$ $\tau_{02}^{*}\left(S_{f_{3}}\right)=0$, whence $\Delta^{n}\left(\operatorname{cl} f_{2}\right)=\Delta^{n}\left(\operatorname{cl} f_{3}\right)=0$. Finally, for $f_{4}=\left(\mathrm{O}_{4}, \gamma \beta a^{k-1}, \mathrm{O}_{3}\right)$, we immediately obtain $S_{f_{4}}=0$. Consequently, $\Delta^{n}=0$ in this case.

2) Assume that $n \equiv 2(\bmod 6)(n \geq 4)$, and put $f \in \operatorname{Ker} \delta_{\mathcal{X} \bullet}^{n}$. Using Corollary 4.14 again, we may assume that $f$ is one of the elements in (4.19), (4.23). Now the differential $d_{n}^{Q} \bullet$ has the form

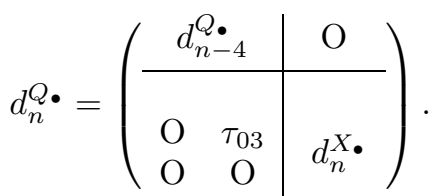

Then

$$
\delta^{n}(\tilde{f})=\left(\mathrm{O}, \tau_{03}^{*}\left(S_{f}\right), \mathrm{O}_{8}\right) .
$$

As above, we see that $\tau_{03}^{*}\left(S_{f}\right)=0$ for all $f$ that represent elements of the above-mentioned basis of the vector space $\mathrm{H}^{n}\left(\mathcal{X}^{\bullet}\right)$; hence, in this case again we have $\Delta^{n}=0$.

3) Assume that $n \equiv 3(\bmod 6)(n \geq 4)$, and put $f \in \operatorname{Ker} \delta_{\mathcal{X}}^{n}$. Using Corollary 4.14, we may assume that $f$ is one of the elements in (4.20), (4.21). Now the differential $d_{n}^{Q}$ • has the form

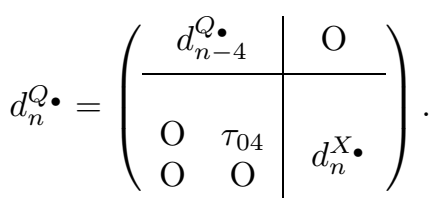

Then

$$
\delta^{n}(\tilde{f})=\left(\mathrm{O}, \tau_{04}^{*}\left(S_{f}\right), \mathrm{O}_{8}\right) .
$$

As above, we see that $\Delta^{n}(\mathrm{cl} f)=0$ for all $f$ that represent elements of the basis of the vector space $\mathrm{H}^{n}\left(\mathcal{X}^{\bullet}\right)$ that was described above for this case, whence $\Delta^{n}=0$.

4) If we take into account the 2-periodicity of the subcomplex $\mathcal{Y}^{\bullet}$ and the 3 -periodicity of the subcomplex $\mathcal{Z}^{\bullet}$ (see Remark 4.12), we see that the above calculation proves also that $\Delta^{n}=0$ for the remaining $n$ (namely, for $n \equiv 4,5$, or $\left.0(\bmod 6)\right)$.

Now relation (4.24) follows from the exactness of the sequence (4.25), with the use of Proposition 4.13 and Corollary 4.16.

Consequently, Theorem 2.1 is proved completely.

Corollary 4.17. For any $n \geq 13$, we have

$$
\operatorname{dim}_{K} \mathrm{HH}^{n}(R)-\operatorname{dim}_{K} \mathrm{HH}^{n-12}(R)=14 .
$$

Proof. Relation (4.26) is deduced from (4.24) with the help of the corresponding analysis of several cases. 


\section{REFERENCES}

[1] K. Erdmann, Blocks of tame representation type and related algebras, Lecture Notes in Math., vol. 1428, Springer-Verlag, Berlin, 1990. MR.1064107

[2] Th. Holm, Derived equivalence classification of algebras of dihedral, semidihedral, and quaternion type, J. Algebra 211 (1999), 159-205. MR 1656577

[3] A. I. Generalov and H. Yu. Kosovskaya, Hochschild cohomology for algebras of dihedral type. IV. The family $D(2 \mathcal{B})(k, s, 0)$, Zap. Nauchn. Sem. S.-Peterburg. Otdel. Mat. Inst. Steklov (POMI) 423 (2014), 67-104; English transl., J. Math. Sci. (N.Y.) 209 (2015), no. 4, 522-548.

[4] F. M. Bleher, Dihedral blocks with two simple modules, Proc. Amer. Math. Soc. 138 (2010), no. 10, 3467-3479. MR2661547

[5] A. I. Generalov, Hochschild cohomology of dihedral type algebras. I. The family D(3K) in characteristic 2, Algebra i Analiz 16 (2004), no. 6, 53-122; English transl., St. Petersburg Math. J. 16 (2005), no. 6, 961-1012. MR 2117449

[6] Hochschild cohomology of dihedral type algebras. II. Local algebras, Zap. Nauchn. Sem. S.-Peterburg. Otdel. Mat. Inst. Steklov. (POMI) 375 (2010), 92-129; English transl., J. Math. Sci. (N.Y.) 171 (2010), no. 3, 357-379. MR2749277

[7] Hochschild cohomology of algebras of dihedral type. III. Local algebras in characteristic 2, Vestn. St. Peterburg Univ. Ser. 1. Mat. Mekh. Astronom. 2010, vyp. 1, 28-38. (Russian) MR2662406 (2011e:16012)

[8] A. I. Generalov, I. M. Zilberbord, and D. B. Romanova, Hochschild cohomology for algebras of dihedral type. V. The family $D(3 \mathcal{K})$ in characteristic different from 2, Zap. Nauchn. Sem. S.-Peterburg. Otdel. Mat. Inst. Steklov. (POMI) 430 (2014), 74-102. (Russian)

[9] A. I. Generalov, Hochschild cohomology of algebras of semidihedral type. I. Group algebras of semihedral groups, Algebra i Analiz 21 (2009), no. 2, 1-51; English transl., St. Petersburg Math. J. 21 (2010), no. 2, 163-201. MR2549450

[10] - Hochschild cohomology of algebras of semidihedral type. II. Local algebras, Zap. Nauchn. Sem. S.-Peterburg. Otdel. Mat. Inst. Steklov (POMI) 386 (2011), 144-202; English transl., J. Math. Sci. (N.Y.) 180 (2012), no. 3, 278-314. MR2784134

[11] Hochschild cohomology of algebras of semidihedral type III. The family $S D(2 \mathcal{B})_{2}$ in characteristic 2, Zap. Nauchn. Sem. S.-Peterburg. Otdel. Mat. Inst. Steklov. (POMI) 400 (2012), 133-157; English transl., J. Math. Sci. (N.Y.) 192 (2013) no. 2, 200-214. MR3029568

[12] _ Hochschild cohomology of algebras of semidihedral type. IV. The cohomology algebra for the series $S D(2 \mathcal{B})_{2}(k, t, c)$ in the case where $c=0$, Zap. Nuchn. Sem. S.-Peterburg. Otdel. Mat. Inst. Steklov. (POMI) 413 (2013), 45-92; English transl., J. Math. Sci. (N.Y.) 202 (2014), no. 3, 360-394. MR3073058

[13] _ Hochschild cohomology of quaternion type algebras. I. Generalized quaternion groups, Algebra i Analiz 18 (2006), no. 1, 55-107; English transl., St. Petersburg Math. J. 18 (2007), no. 1, 37-76. MR2225213

[14] A. I. Generalov, A. A. Ivanov, and S. O. Ivanov, Hochschild cohomology of quaternion type algebras. II. The family $Q(2 \mathcal{B})_{1}$ in characteristic 2, Zap. Nauchn. Sem. S.-Peterburg. Otdel. Mat. Inst. Steklov (POMI) 349 (2007), 53-134; English transl., J. Math. Sci. (N.Y.) 151 (2008), no. 3, 2961-3009. MR 2742854

[15] A. I. Generalov, Hochschild cohomology of quaternion type algebras. III. Algebras with a small parameter, Zap. Nauchn. Sem. S.-Peterburg. Otdel. Mat. Inst. Steklov (POMI) 356 (2008), 46-84; English transl., J. Math. Sci. (N.Y.) 156 (2009), no. 6, 877-901. MR2760365

[16] A. A. Ivanov, Hochschild cohomology of algebras of quaternion type: the family $Q(2 \mathcal{B})_{1}(k, s, a, c)$ in characteristic different from 2, Vestn. St. Peterburg. Univ. Ser. 1. Mat. Mekh. Astronom. 2010, vyp. 1, 63-72. (Russian) MR2662411(2011f:16021)

[17] K. Erdmann, Th. Holm, and N. Snashall, Twisted bimodules and Hochschild cohomology for selfinjective algebras of class $A_{n}$. II, Algebr. Represent. Theory 5 (2002), no. 5, 457-482. MR1935856

[18] A. I. Generalov and M. A. Kachalova, Bimodule resolution of the Möbius algebra, Zap. Nauchn. Sem. S.-Peterburg. Otdel. Mat. Inst. Steklov. (POMI) 321 (2005), 36-66; English transl., J. Math. Sci. (N.Y.) 136 (2006), no. 3, 3850-3866. MR2138411

[19] M. A. Kachalova, The Hochschild cohomology for the Möbius algebra, Zap. Nauchn. Sem. S.Peterburg. Otdel. Mat. Inst. Steklov. (POMI) 330 (2006), 173-200; English transl., J. Math. Sci. (N.Y.) 140 (2007), no. 5, 699-715. MR2253573

[20] M. A. Pustovykh, The Hochschild cohomology ring of the Möbius algebra, Zap. Nauchn. Sem. S.Peterburg. Otdel. Mat. Inst. Steklov. (POMI) 388 (2011), 210-246; English transl., J. Math. Sci. (N.Y.) $\mathbf{1 8 3}$ (2012), no. 5, 692-714. MR2822523 
[21] Yu. V. Volkov and A. I. Generalov, Hochschild cohomology for self-injective algebras of tree class $D_{n}$. I, Zap. Nauchn. Sem. S.-Peterburg. Otdel. Mat. Inst. Steklov. (POMI) 343 (2007), 121-182; English transl., J. Math. Sci. (N.Y.) 147 (2007), no. 5, 7042-7073. MR2469415

[22] Yu. V. Volkov, Hochschild cohomology for self-injective algebras of tree class $D_{n}$. II, Zap. Nauchn. Sem. S.-Peterburg. Otdel. Mat. Inst. Steklov. (POMI) 365 (2009), 63-121; English transl., J. Math. Sci. (N.Y.) 161 (2009), no. 4, 492-524. MR2749135

[23] Yu. V. Volkov and A. I. Generalov, Hochschild cohomology for self-injective algebras of tree class $D_{n}$. III, Zap. Nauchn. Sem. S.-Peterburg. Otdel. Mat. Inst. Steklov. (POMI) 386 (2011), 100-128; English transl., J. Math. Sci. (N.Y.) 180 (2012), no. 3, 252-268. MR2784132

[24] Yu. V. Volkov, Hochschild cohomology for nonstandard sef-injective algebras of free class $D_{n}$, Zap. Nauchn. Sem. S.-Peterburg. Otdel. Mat. Inst. Steklov. (POMI) 388 (2011), 48-99; English transl., J. Math. Sci. (N.Y.) 183 (2012), no. 5, 600-628. MR2822516

[25] _ The Hochschild cohomology algebra for a family of self-injective algebras of tree class $D_{n}$, Algebra i Analiz 23 (2011), no. 5, 99-139; English transl., St. Petersburg Math. J. 23 (2012), no. 851-879. MR2918425

[26] _ Hochschild cohomology for self-injective agebras of tree class $D_{n}$. IV, Zap. Nauchn. Sem. S.-Peterburg. Otdel. Mat. Inst. Steklov. (POMI) 388 (2011), 100-118; English transl., J. Math. Sci. (N.Y.) 183 (2012), no. 5, 629-639. MR2822517

[27] _ Hochschild cohomology for self-injective algebras of tree class $D_{n}$. V, Zap. Nauchn. Sem. S.-Peterburg. Otdel. Mat. Inst. Steklov. (POMI) 394 (2011), 140-173; English transl., J. Math. Sci. (N.Y.) 188 (2013), no. 5, 551-569. MR2870173

[28] M. A. Pustovykh, The Hochschild cohomology ring for self-conjective algebras of tree class E ${ }_{6}$, Zap. Nauch. Sem. S.-Peterburg. Otdel. Mat. Inst. Steklov. (POMI) 423 (2014), 205-243; English transl. J. Math. Sci. (N.Y.) 209 (2015), no. 4, 614-644.

[29] A. I. Generalov and N. Yu. Kosovskaya, The Hochschild cohomology of Liu-Schulz algebras, Algebra i Analiz 18 (2006), no. 4, 39-82; English transl., St. Petersburg Math. J. 18 (2007), no. 4, 539-572. $\operatorname{MR} 2262583$

[30] Th. Holm, Hochschild cohomology of tame blocks, J. Algebra 271 (2004), no. 2, 798-826. MR2025551

[31] C. T. C. Wall, Resolutions for extensions of groups, Proc. Cambridge Phil. Soc. 57 (1961), 251-255. MR 0178046

[32] Yu. V. Volkov, A. I. Generalov, and S. O. Ivanov, On the construction of bimodule resolutions with the help of the Happel lemma, Zap. Nauchn. Sem. S.-Peterburg. Otdel. Mat. Inst. Steklov. (POMI) 375 (2010), 61-70; English transl., J. Math. Sci. (N.Y.) 171 (2010), no. 3, 338-343. MR.2749275

[33] A. I. Generalov, Cohomology of algebras of dihedral type. IV: The family D(2B), Zap. Nauchn. Sem. S.-Peterburg. Otdel. Mat. Inst. Steklov. (POMI) 289 (2002), 76-89; English transl., J. Math. Sci. (N.Y.) 124 (2004), no. 1, 4719-4726. MR1949735

Department of Mathematics and Mechanics, St. Petersburg State University, UniversitetSkil Pr. 28, Petrodvorets, St. Petersburg 198504, Russia

E-mail address: ageneralov@gmail.com

School no. 642 "Earth and Universe", Gavanskaya ul. 54, St. Petersburg 199406, Russia

E-mail address: dashh@@gmail.com

Received 10/JUNE/2015 